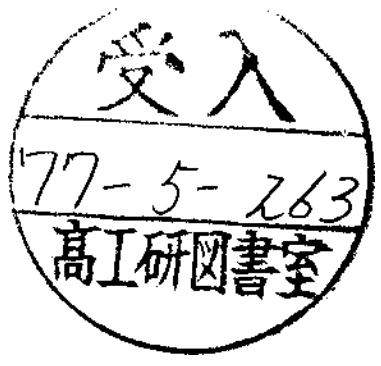

EUROPEAN ORGANIZATION FOR NUCLEAR RESEARCH

CERN ISR-VA/77-23

\title{
ISR CLEARING CURRENT MONITORING SYSTEM
}

by

0 . Gröbner and P. Strubin

Presented at the 1977 Particle Accelerator Conference, Chicago, March 16-18, 1977

Geneva, Switzerland

March 1977 
CONTENTS

SUMMARY

1. INTRODUCTION

2. CLEARING CURRENT DUE TO PRIMARY IONISATION

3. THE MONITORING SYSTEM

4. CLEARING CURRENT EXPERIMENTS

REFERENCES 


\title{
ISR CLEARING CURRENT MONITORING SYSTEM
}

\author{
0 . Gröbner and P. Strubin \\ CERN \\ Geneva, Switzerland
}

\section{Surmary}

Clearing electrodes remove electrons produced by ionisation of the residual gas in the ISR. The clearing current from a section of length $L$ is related to the beam current $I$ and the absolute pressure $F$ by $I_{C}=0.76 \mathrm{I} \mathrm{L} \mathrm{P.} \mathrm{The} \mathrm{described} \mathrm{monitoring} \mathrm{system} \mathrm{meas-}$ ures clearing currents from over 300 electrodes with a sensitivity of $20 \mathrm{pA}$, adequate to observe pressure changes of $510^{-14}$ Torr. As compared with conventional UHV gauges which measure only locally, clearing currents yield the integrated pressure along the beam. For maximum flexibility and to cope with future extensions, the control and data acquisition system has been designed around a microprocessor unit connected through an existing CAMAC link to the central ISR computer. Measurements have been performed of the pressure stability in the presence of high intensity beams and of the secondary ionisation rate by electrons trapped in local neutralization pockets. At several occasions, large clearing currents were observed which have been explained tentatively by ionisation of metal atoms sputtered from the vacuum chamber.

\section{Introduction}

The ISR are equipped with electrostatic electrodes mounted above and below the beam in the coil overhangs of the bending magnets to clear electrons produced in ionising collisions between stored high energy protons and the rest gasl. Following the discovery of the coupled electron-proton oscillations ${ }^{2}$ which strongly affect the lifetime and blow-up rate of the beam, causing increased background for colliding beam experiments, the clearing system - initially designed to guarantee a small neutralisation tune shift - had to be improved to reduce the electron concentration below the e-p instability threshold. Mainly localised electron pockets, occurring at the maxima of the longitudinal variations of the beam potential, contribute to the residual neutralization; to clear them efficiently many additional electrodes have been installed (at present more than 300 pairs per ring).

Optimum clearing up to the highest beam currents achieved in the ISR require electric fields of the order of $10^{5} \mathrm{~V} / \mathrm{m}$, corresponding to a potential difference between plates of about $6 \mathrm{kV}$. To clear electrons drifting in longitudinal direction, the difference between the beam potential in the circular pipe and the near-elliptic cross-section formed by the electrodes is compensat ed by assymmetric potentials of about +6 and $0.2 \mathrm{kV}$ on opposite plates.

The electrodes of an octant and of the same potential are powered from a common high voltage supply. The clearing voltage is maintained constant irrespective of the stored beam current. The power supplies provide a monitoring facility for the sum clearing currents from individual octants. This information has proven to be very valuable, in particular when operating the ISR at high beam currents close to the vacuum stability limit. Since the clearing current depends on the pressure, a beam induced pressure rise is readily detectable. How ever, the localisation which can be achieved is insufficient; therefore, a new system has been developed and is presently under construction for measuring the current of individual electrodes.

\section{Clearing Current Due to Primary Ionisation}

Electrons created by ionisation of rest gas molecules by high energy protons contribute to the clearing current apart from a fraction which receives sufficient energy to overcome the positive space charge potential of the beam (typically increasing by $100 \mathrm{~V} / \mathrm{A}$ of beam) and escapes to the vacuum chamber. Measurements suggest that most electrons have, in fact, energies of less than $50 \mathrm{eV}$. Hence, the number which can escape decreases rapidly with increasing beam current and can be neglected above about $1 \mathrm{~A}$.

The production rate of electrons per proton in the rest gas of density $\mathrm{n}$ with the ionisation cross-section $\sigma$ is $R=\sigma c n$, $c$ being the velocity of light. In a mixture of gases, the contribution of the partial pressures has to be known. For the ISR, the average composition after glow discharge cleaning and $300^{\circ} \mathrm{C}$ bakeout is listed below. Cross-sections have been calculated using experimental data obtained with high energy electrons and positrons ${ }^{3}$. Since for ISR energies from 11 to $31 \mathrm{GeV}$ the cross-sections increase by less than $10 \%$, this energy dependence is neglected.

\begin{tabular}{|l|c|c}
\hline Gas & $\begin{array}{c}\text { Average fraction } \\
\text { in the ISR }\end{array}$ & $\begin{array}{c}\sigma \text { in units of } 10^{-23} \mathrm{~m}^{2} \\
\text { for } 26 \mathrm{GeV} \text { protons }\end{array}$ \\
\hline $\mathrm{H}_{2}$ & 0.935 & 1.8 \\
$\mathrm{CH} 4$ & 0.014 & 9.7 \\
$\mathrm{CO}, \mathrm{N}_{2}$ & 0.036 & 8.2 \\
$\mathrm{CO}_{2}$ & 0.015 & 13 \\
\hline
\end{tabular}

The clearing current from a section of length $L$ is obtained by

$$
I_{C}=0.76 \mathrm{~L} \mathrm{I} \mathrm{P,}
$$

$\mathrm{P}$ being the absolute pressure in Torr and $\mathrm{L}$ in metre. Expressed in nuclear scattering pressure $\mathrm{P}_{\mathrm{NS}}$, relevant for the beam decay, the numerical factor is 4.75. (For the average gas composition in the ISR, $P_{N S}=0.16 \mathrm{P}$. ) Within the errors of the measurements, the nuclear scattering pressure determined from clearing currents is consistent with the observed decay rates of low loss rate beams and agrees with the average reading of the UHV gauges.

\section{The Monitoring System}

The monitoring system for individual electrodes and providing increased spacial resolution has been designed for the following applications:

- to measure the integrated pressure along the beam as opposed to the local reading from UHV gauges;

- to measure electrons created by proton losses against the vacuum chamber to localise regions of abnormal losses or aperture restrictions;

- to localise neutralization pockets which cause e-p instabilities;

- to study clearing currents due to effects of secondary ionisation.

The sensitivity has been defined for a minimum length $\mathrm{L}=2.5 \mathrm{~m}$ (short magnet unit), a nuclear scattering pressure of $10^{-12}$ Torr and a 2 A circulating beam. According to equation ( 1 ), the clearing current is about $210^{-11} \mathrm{~A}$. Since the current increases with beam intensity, pressures in the $10^{-14}$ Torr range can be detected with a stack of $25 \mathrm{~A}$. The upper limit, more arbitrarily 
chosen at $10^{-6}$, defines a dynamic range for the system of 5 decades.

Despite the fact that the clearing currents are two orders of magnitude higher than typical ionisation gauge signals, the technical difficulty consists of measuring at a high voltage. The adopted measuring principle which uses the existing parts of the RF-termination of the electrodes is shown in Fig. 1. During a measurement, the high voltage relay in series with the decoupling resistor $R_{d}$ isolates the electrode from the HV network. The clearing current discharges the d.c. blocking capacitor $(\mathrm{C}=470 \mathrm{pF})$ and is measured by the electrometer.

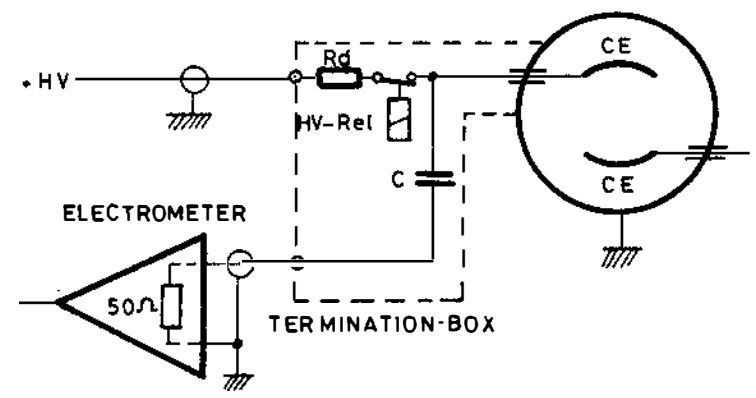

Fig. 1 Principle of Current Measurement on a Clearing Electrode.

Electrometer Amplifier - The current signal is fed to the electrometer via a low noise triaxial cable. The amplifier input is normally shunted by a field effect transistor to obtain a matched high frequency termination up to about $480 \mathrm{MHz}$ (Fig. 2). During a measurement, this transistor is driven to its non-conducting state. The amplifier operates as a current to voltage converter with switchable gains of $10^{7} \mathrm{~V} / \mathrm{A}$ and $10^{5} \mathrm{~V} / \mathrm{A}$. Special design efforts in view of radiation damage have been made to reduce the component number to a minimum. A careful selection of components and printed circuit board design ensures leakage and offset currents typically below $10^{-11}$ A. Overall accuracy is about $5 \%$ of full scale.

Signal Acquisition - Each electrometer is powered by an individual supply to avoid ground loop problems. These supplies are located in an auxiliary unit which also contains a CMOS-switch array to select individual electrometer outputs (see Fig. 3 ).

To increase noise immunity, the controls of the switches and of the electrometer functions are driven through opto-couplers. The switching functions and ana-

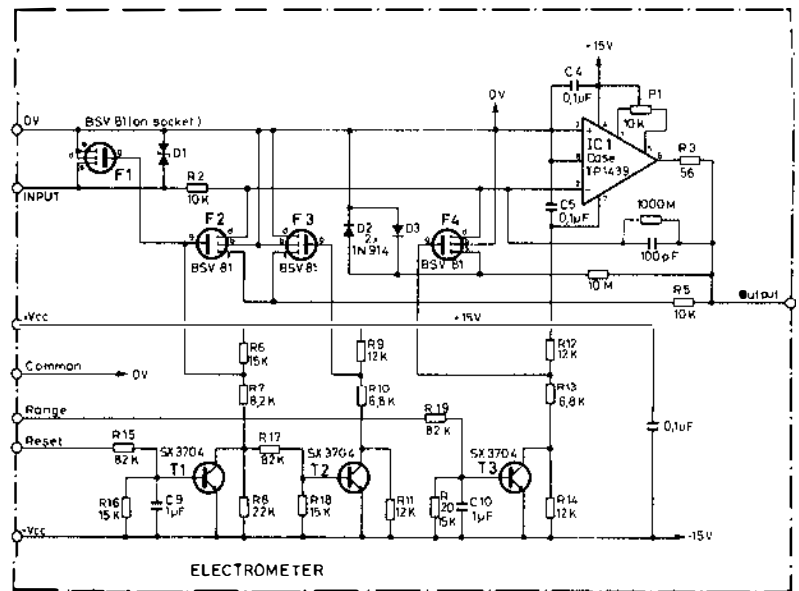

Fig. 2 Electrometer Amplifier with Switchable Gain of $10^{7}$ and $10^{5} \mathrm{~V} / \mathrm{A}$.

log signal lines operate in differential mode. A 12-pole cable connects the multiplexing units to a main control unit outside the ISR tunnel. Sharing this cable between analog and digital signals has been found acceptable since the latter signals do not change state during a measuring cycle.

Control System - One main controller drives four auxiliary units in each octant. A 12-bit analog to digital converter with built-in multiplexer allows data acquisition. Its resolution matches the typical leakage currents of the electrometers. The control unit is built around an 8-bit micro-processor with some 1500 words of program memory and 500 words of read/write memory for temporary storage. A 24-bit output register and an 8-bit input register allow the dialog with the central computer in the control room through an existing CAMAC link.

The main tasks of this system are the following:

- Automatic scanning of clearing currents. At a measuring time per electrode of about $100 \mathrm{~ms}$, an octant may be scanned in less than $5 \mathrm{~s}$. Measurements in different octants can proceed independently. Figure 4 shows the pressure profiles along ISR Sector 31 obtained at beam currents of 10 and $39 \mathrm{~A}$.

- Perform synchronised measurements on selected electrodes, e.g. to localise beam losses during injection.

- Calculate an apply offset and leakage current corrections for each electrode.

- Facilitate the maintenance of the clearing system. $\begin{array}{ll}\text { ELECTROMETER } & \text { AUX. MULTIPLEXING UNIT } \\ & \text { AND ELECTROMETER SUPPLY }\end{array}$

MAIN CONTROL UNIT

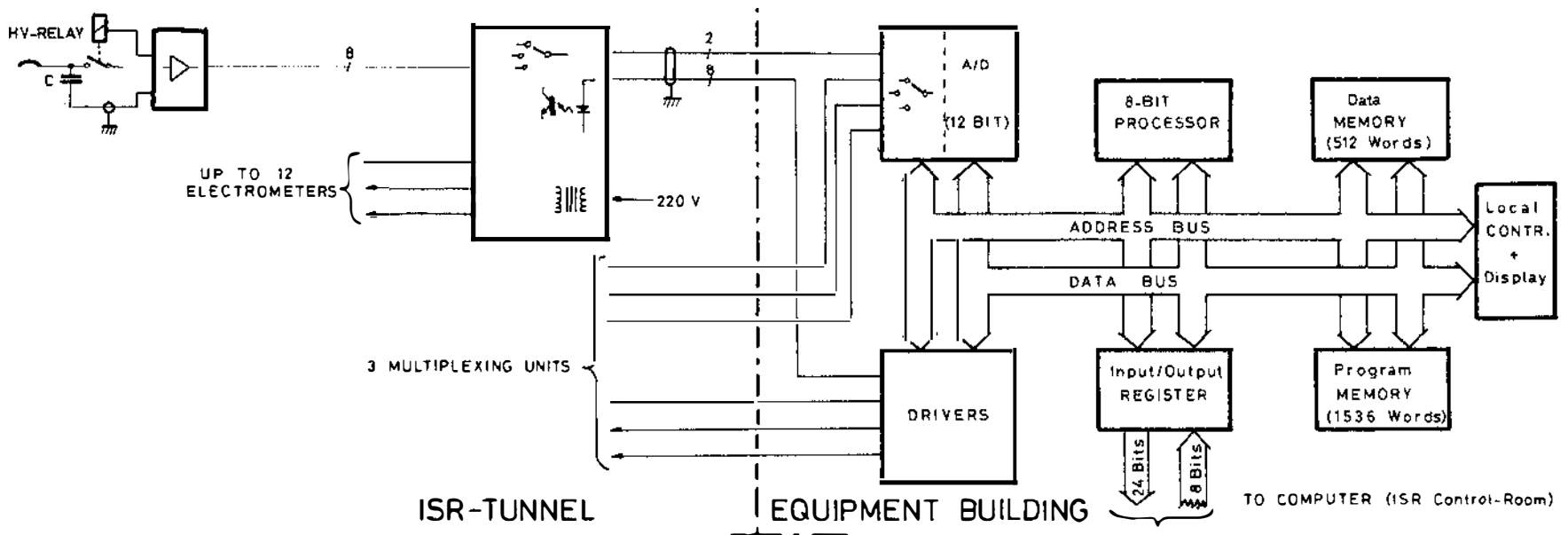

Fig. 3 System Block Diagram for Data Acquisition and Electrometer Controls 


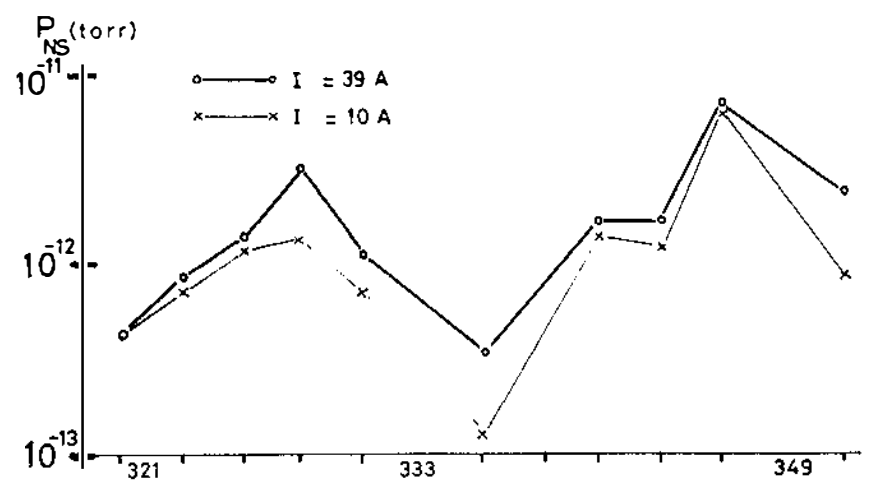

Fig. 4 Pressure Profiles in ISR Sector 31 Measured at Beam Intensities of $10 \mathrm{~A}$ and $39 \mathrm{~A}$ and Showing Local Beam Induced Pressure Rises.

\section{Clearing Current Experiments}

The space charge potential of the beam and hence the required clearing voltage do not increase linearly with current. Figure 5 shows the beam potential as a function of current. The curve has been calculated for a rectangular geometry ${ }^{4}$.

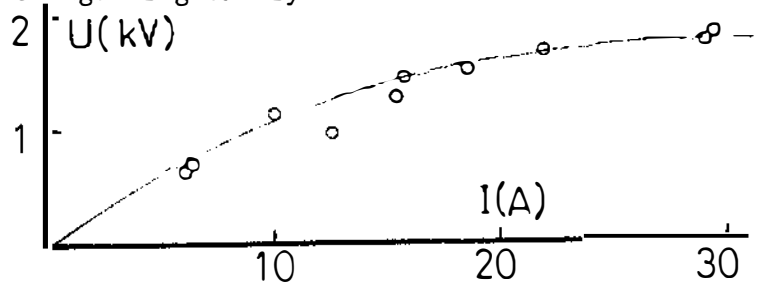

Fig. 5 Beam Potential versus Stacked Current as Determined from the Clearing Electrode Potential at Saturated Electron Current.

Secondary production of electrons increases with the residual gas density and the time needed for clearing primary electrons $t_{c}$. The clearing current observed in a neutralization pocket is

$$
I_{c}=I_{C, p r i m}(1+\rho) \text {, }
$$

where $\rho$ is a secondary ionisation coefficient. Since electrons gain energy gradually in multiple Coulomb col1 isions with protons, the clearing time $t_{c}$ can be calculated from the rate of energy gain and the depth of the potential well. Figure 6 shows $\rho$ as a function of $t_{c}$ at a pressure of $1.5 \quad 10^{-11}$ Torr corresponding to $R=810^{-3} \mathrm{~s}^{-1}$. Secondary production is negligible at low pressure and when the clearing system is effective $\left(t_{c} \leq 10^{-3} \mathrm{~s}\right)$. Electron pockets which participate in coupled electron-proton oscillations may be detected by the pulsing clearing current due to the successive build-up of the instability.

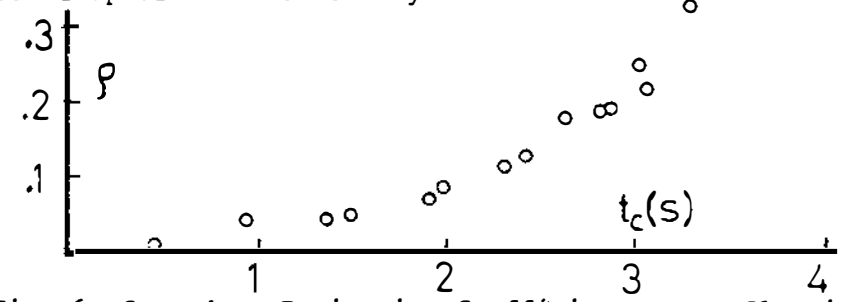

Fig. 6 Secondary Ionisation Coefficient $\rho$ vs. Clearing Time $t_{c}$ in $s . \quad t_{c}$ is Calculated from Energy Transfer by Multiple Coulomb Collisions with Protons.

Ionisation of sputtered metal atoms - Clearing current measurements have frequently shown a production rate increasing with beam intensity at apparently constant pressure (Fig. 7). This effect has been explained tentatively by ionisation of sputtered metal atoms un- detected by ionisation gauges, because of their sticking probability. Sputtered atoms of density $\mathbf{N}$ and ionisation cross-section $\sum$ give rise to a clearing current per unit length of

$$
I_{\mathrm{c}}=\left(\sigma \mathrm{n}+\sum \mathrm{N}\right) \mathrm{I} \text {. }
$$

The density of sputtered atoms in a chamber of radius $r$ follows from

$$
\mathrm{r}^{2} \pi \frac{\mathrm{dN}}{\mathrm{dt}}=\frac{\alpha \sigma I}{\mathrm{e}} \mathrm{n}+\frac{\mathrm{A} \Sigma \mathrm{I}}{\mathrm{e}} \mathrm{N}-\frac{\pi}{2} \overline{\mathrm{v}} \mathrm{sr} \mathrm{N},
$$

where $\alpha$ and $A$ are the sputtering coefficients for the residual gas molecules and the metal atoms, respectively, $e$ is the unit charge, $\bar{v}$ the mean velocity and $s$ the sticking probability of the sputtered atoms. In equilibrium

$$
\overrightarrow{\mathrm{N}}=\frac{\alpha \sigma \mathrm{I}}{\pi / 2 \text { eṽs }-\mathrm{A} \Sigma \mathrm{I}} \mathrm{n},
$$

and the resulting clearing current

$$
I_{c}=\sigma I n\left(1+\frac{\alpha \Sigma I}{\pi / 2 \operatorname{evvr}-\mathrm{A} \Sigma I}\right) \text {. }
$$

Because sputtering increases with $X$ but re-adasorption remains constant, a limiting current exists for which the denomination vanishes.

The interpretation of excess currents by metal sputtering leads - for reasonable values of the assumed $A$ and $\Sigma$ - to sticking coefficients of only $10^{-2}$ to $10^{-3}$. There is no information available for metal atoms sticking on the ISR stainless steel vacuum chamber, but neasurements of sodium condensation have produced similar results ${ }^{5}$. So far, there is little evidence that metal sputtering would limit the beam intensity in the ISR.

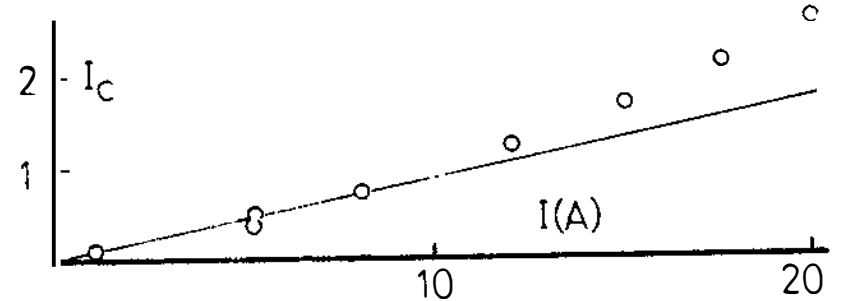

Fig. 7 Clearing Current Ic (arbitrary units) vs. Beam Current in Ampère Showing Deviation from the Expected Linear Dependence at Constant Residual Pressure Attributed to Sputtered Metal Atoms.

\section{References}

1. B. Angerth, E. Fischer, 0. Gröbner, The clearing fields of the ISR, VIIIth Int. Conf. on High Energy Accelerators, CERN (197I).

2. H.G. Hereward, Coherent instability due to electrons in a coasting proton beam, CERN Report 71-15 (1971).

3. F.F. Ricke and W. Prepejchal, Ionisation cross-sections of gaseous atoms and molecules for high energy electrons and positrons, Phys. Rev. A, Vol. 6, No. 4 (Oct. 1972).

4. 0. Gröbner, K. Hïbner, Computation of the electrostatic beam potential in vacuum chambers of rectangular cross-section, Div. Report CERN ISR-TH-VA/75-27 (1975).

5. B. Vosicki, K. Zankel, Generator of a dense atomic gas curtain, Journal of Physics E, Scientific Instruments 1975, Vol. 8 (1975). 